\title{
COMMENT
}

Received 11 Apr 2016 | Accepted 11 May 2016 | Published 7 Jun 2016

\section{Stretching the boundaries: language learning psychology}

\author{
Sarah Mercer $^{1}$ and Stephen Ryan ${ }^{2}$
}

\begin{abstract}
In this article, we trace the story of a newly emerging field of study and the role of interdisciplinarity in its development. As the name suggests, the psychology of language learning is a field that connects the two disciplines of psychology and language learning, but it also encompasses other disciplines as diverse as communication studies, education studies and cultural studies. As a recognizable, independent field of enquiry with an established community of scholars, it is still in its infancy. However, its short history reveals key issues relating to the nature of interdisciplinarity and the struggles of a new interdiscipline to emerge and gain acceptance. We hope that this article will simultaneously serve as a call to like-minded scholars in our field to adopt a more overtly interdisciplinary approach and as a plea for recognition and support within the established community of interdisciplinarians. Although our core narrative may be a familiar one to expert interdisciplinarians, we still believe that the specifics of our account represent an original contribution to understanding the processes of an emerging interdiscipline. To help position this article and the discussions within, we will begin with a brief overview of the development of the field, discussing some of the reasons behind its emergence and identifying key characteristics. We will then discuss the attractions of such an interdisciplinary field of research and reflect on the challenges facing those working in this area. We will conclude by proposing an agenda to help promote future interdisciplinary research into the psychology of language learning. This article is published as part of a collection on interdisciplinarity.
\end{abstract}

\footnotetext{
${ }^{1}$ University of Graz, Graz, Austria ${ }^{2}$ Waseda University, Tokyo, Japan Correspondence: (e-mail: sarah.mercer@uni-graz.at)
} 


\section{The emergence of the study of psychology in language learning}

he emergence of the field of psychology in foreign language learning is perhaps best understood within the context of the development of its parent discipline, applied linguistics. The roots of applied linguistics lie firmly in the field of language education, but as the field has grown and expanded, it has moved away from this narrow educational base, extending its remit to cover "problems in the world in which language is implicated" (Cook, 2003: 3). Many language teachers have been sceptical of the practical value of much applied linguistics research. One reason for this suggests Widdowson (2006) is the implied primacy of "linguistics", which teachers may not regard as particularly relevant to learning contexts. Instead, there has been a growing awareness of a need for academic enquiry with a more pronounced focus on issues connected to learning processes and the individuals involved, as opposed to the focus on language per se.

In terms of both practice and research, language education has been moving away from solely language or teaching-based models towards a greater focus on the various contributions learners make to their own learning (Breen, 2001) as evident in the shift towards more learner-centred models of learning. In empirical terms, investigations of how learners contribute to their own learning have often largely been concerned with gaining an understanding of why certain individuals are able to learn languages more successfully than others, considering in particular how mental processes specific to individuals affect learning outcomes. This interest in the role of individual mental processes has inevitably led some scholars in the direction of educational psychology, with the aim of exploring how established concepts from educational psychology can contribute to a fuller understanding of life in the language classroom. In effect, this represents a realignment of language education with mainstream school subjects. For much of its relatively short history, foreign language education has set itself apart-and has been set apart-from other school subjects, and an unfortunate consequence has been that language education has often not made full use of the findings of mainstream education research and educational psychology. However, the emerging field of language learning psychology aims to go beyond merely drawing on insights from an external field; we hope to build something unique, something informed by a range of perspectives but not dominated by any single disciplinary outlook.

The growth and course of the development of this newly emerging interdiscipline can be plotted through the publication of several key books in the field. The initial seeds of this movement can be traced back to the publication of McDonough's (1981) publication Psychology in Foreign Language Teaching. However, the field gained momentum and direction with Williams and Burden's (1997) Psychology for Language Teachers. This book created the template for a future interdisciplinary approach in that it was a highly original, successful collaboration between a language educator and an educational psychologist; in effect, we can identify the beginnings of our interdiscipline in these two scholars' interdisciplinary curiosity and collaboration. Things really began to take off with Dörnyei's (2005) The Psychology of the Language Learner: Individual Differences in Second Language Acquisition. The significance of this landmark publication is implicit in its title. Previous to the publication of this book, the study of individual variation in language learning was very much the preserve of the field of individual differences in second language acquisition, an area primarily concerned with isolating key variables, most notably aptitude and motivation, and measuring their effects on learning outcomes. However, in this particular book, Dörnyei-perhaps the most important individual driving the development of this field of study-signals a move away from the established individual differences paradigm, in which he established his reputation, towards a fuller, more rounded account of the language learner as a real person, as opposed to a composition of abstracted measurable variables, by foregrounding the word "psychology" in the title. In 2009, Dörnyei developed some of his earlier ideas in a highly ambitious work, The Psychology of Second Language Acquisition. In the preface to this volume, Dörnyei identifies a fundamental challenge facing scholars looking to research this area, positioning himself as an outsider looking in on two separate academic disciplines. In effect, Dörnyei was arguing that to investigate something as complex as the psychology of foreign language learning, it is necessary to step outside the confines of a single discipline and construct a more comprehensive understanding. It is possible that his perceptions of outsider status were a reflection of the struggles of a de facto interdisciplinarian to accommodate himself within an environment that values disciplinary purity. Dörnyei's influence has been so powerful that this narrative of "outsider" has been broadly accepted with little attempt by scholars to contextualize developments in terms of the emergence of an interdiscipline.

A further significant subnarrative within Dörnyei's (2009) book is how he-a writer best known for his work on motivationdeals with this problematic concept; motivation research has long subsumed research into other areas of language learner psychology (see Boo et al., 2015), but with this publication, Dörnyei makes an explicit effort to situate motivation as one part of a broader psychology of language learning. Picking up the challenge of integrating various aspects of language learner psychology, Mercer et al. (2012) edited a volume that brought together a number of key scholars who had been pursuing apparently disparate lines of investigation, but all broadly relating to psychology in language learning. The various contributions explored some of the commonalities and interconnections among what had up to that point been considered discrete areas of research and the book provided the foundations for the first international conference held in Graz (2014) devoted to research into psychology in language learning; the second already organized for August 2016. The momentum created by that first conference contributed to subsequent publication surge (for example, Ryan and Mercer, 2015a, b; Gkonou et al., 2015), but perhaps most significant has been Williams et al.'s (2015) Exploring Psychology in Language Learning and Teaching, which marked a move into mainstream language teacher education, and Dörnyei and Ryan's (2015) The Psychology of the Language Learner Revisited. This last volume represents both an update and challenge to the basic premise of Dörnyei's highly influential 2005 book and indicates the end of the dominance of the individual differences paradigm-signalled by the complete omission of the term from the title-and the introduction of new approaches to theory and research.

Indeed, perhaps one way in which language learning psychology is ahead of mainstream educational psychology is the way in which it is much more methodologically diverse because of its dual heritage (cf. MacIntyre and Mercer, 2014). A key defining characteristic of recent research in the field has been a concern with the interactions between various aspects of psychology and also with understanding learners as socially situated beings. This represents a significant break from previous approaches to the earlier study of individual differences, which were rooted in the positivist, quantitative research tradition, and which were almost exclusively concerned with isolating and measuring discrete variables within individuals. Instead, recent research has been more open to qualitative enquiry and methodological plurality, and as a consequence of this methodological shift, there has been a pronounced tendency to favour non-linear accounts of learning 
that shun simplistic cause-effect explanations and that also account for the role of contextual variation.

The new field has grown rapidly through a flurry of publications and conferences into a recognizable community of scholars. At present, the majority of these scholars come from the field of applied linguistics with minimal formal training in the other core discipline, psychology. Yet, there are also some key figures in the field who have trained as psychologists or communication scholars with in turn little or no applied linguistics or TESOL background. On the whole, it is perhaps fair to say that the majority of those working in the field stem from applied linguistics backgrounds, and as such, the parent discipline has had and still does have a pronounced influence on the field. Naturally, this implies a high degree of overlap and commonality, despite the obvious distinct content focus of the area of psychology in language learning.

As newcomers to the literature on theories of interdisciplinarity, it has been highly reassuring, even empowering, for us to observe the degree to which the development of our emerging interdiscipline and its hallmark characteristics are consistent with established theory (Newell, 2013). Our field began with intellectual curiosity and interdisciplinary collaboration exploring the gaps between existing, established disciplines, conforming to the classic fish-scale model (Campbell, 1969). From these tentative origins, researchers gradually became more assertive in their efforts to contextualize psychology in language learning, to describe a much broader picture. Furthermore, other traits that we regard as fundamental to our emerging field, such as the drive to explore areas of commonality and interconnections between disciplines, the need for methodological plurality, and a tendency towards non-linear accounts, we now recognize as core propensities of interdisciplinarity. In place of an essentially negative narrative, based around the struggle to conform to established disciplinary norms, we are now able to portray the development of our field in a much more positive light, as part of broader community exploring the possibilities of integrating elements from established disciplines in ways that offer unique, more comprehensive insights (see Fuchsman, 2012).

\section{Advantages/attractions of interdisciplinarity}

We are aware that for experienced interdisciplinarians there may be sections of this article that read like an account of a belated discovery of the wheel. However, there is another intended audience that may be neither familiar with nor convinced by concepts of interdisciplinarity and the arguments set out in the next two sections are primarily aimed at these readers, especially those within our particular respective fields.

As mentioned earlier, the study of psychology in language learning touches on several disciplines; however, in this article, we will concentrate on the two core fields that lie at its heart, namely, applied linguistics and psychology. We do so because the various areas of commonality and tension between these two disciplines best illustrate both the attractions and challenges facing an interdisciplinary field of study as it seeks to establish its identity and academic legitimacy.

Applied linguistics is a field eager to highlight its own interdisciplinary credentials, one in which "the applied linguist is deliberately eclectic, drawing on any source of knowledge that may illuminate the language problems" (Davies, 2007: 68), although explicit discussions of the nature of that interdisciplinarity or specific reference to theories of interdisciplinarity remain rare (for an authoritative exception, see Widdowson, 2006). We also need to take care not to present applied linguistics as some kind of monolithic homogenous field, since there are many, especially regional, variations. Nevertheless, within the mainstream Anglophone applied linguistics tradition, at least, an interdisciplinary approach is not considered controversial; indeed, it can be considered the default assumption for most. However, in other cultures and countries, applied linguistics may be more narrowly defined and psychology oriented perspectives may still be frowned up or viewed as fringe activities.

Perhaps one of the main reasons behind the growing interest in the psychology of language learning has been the recognition of the need for more focused enquiry into the real-world realities of classroom learning. Classrooms tend to be messy, unpredictable places, and to gain more meaningful insights into what is taking place in those classrooms it is necessary to avoid simplistic or one-dimensional accounts. Instead, more complex interdisciplinary responses are required from a range of methodological and epistemological perspectives. Such interdisciplinary work can have many benefits both for understandings in the field itself as well as the individuals engaged in such studies.

First, working in an interdisciplinary way can lead to greater professional expertise, flexibility and creativity. In an interdisciplinary environment, scholars can learn to become more open to alternative ways of working and researching and, in this way, the experiences in both fields can lead to a valuable cross-fertilization of ideas. Exposure to new ideas is not only intrinsically rewarding for a curious academic, it can also lead to fresh perspectives and insights that can be applied within their home discipline and beyond.

A further benefit of working in an interdisciplinary area is that scholars are more likely to find their central assumptions and their typical modes of thinking or working regularly challenged. Although working in unfamiliar ways is rarely an immediately comfortable experience, it can be enormously invigorating. It is also an approach that can expand horizons, with opportunities to attend a wider range of conferences, or other professional events, and come into contact with new people and fresh ideas. This can lead scholars to challenge their own blind spots and the various assumptions of their respective disciplines, thereby enhancing critical thinking and generating innovative lines of thinking. It may be possible to encourage academics in other disciplines to join interdisciplinary undertakings by highlighting how these innovative lines of thinking may give them a leading edge within their "home" discipline.

Those of us coming from a background where the benefits of interdisciplinarity are assumed need to exercise caution in assuming the same of researchers from other backgrounds. It is highly unrealistic to expect people to join us in creating a truly interdisciplinary field of study simply because we ask. We need to make interdisciplinary collaboration an attractive proposition and a policy of gradual persuasion focusing on the potential benefits that collaborators can apply within their home discipline may be the best way to arouse initial interest.

\section{Challenges of an interdisciplinary approach}

Despite the obvious benefits of an interdisciplinary approach, there are also considerable risks and challenges. These challenges appear at various levels, from the limitations of an individual researcher to deeply entrenched institutional structures and practices.

The strength of any field of human endeavour ultimately lies with the people involved. If an emerging field of study can attract a lively and active community of scholars, it will thrive; if it fails to do so, then its future prospects are bleak. Attracting and engaging new scholars can often depend on everyday issues, such as the need for secure employment with opportunities for career advancement and the need for job satisfaction and peer recognition. Academic 
disciplines represent distinct cultures with their own established norms, shared discourses and shared identities with clear criteria for group membership and participation. For young academics at the beginning of their careers, it is often easier to conform to the norms and expectations of established cultures than to risk isolation and career stagnation in a fringe field of study. It can feel very lonely, isolated and doubtless it is hard to persevere without any sense community or visible group with which to identify. This means that scholars are often reluctant to cross boundaries and work in these interdisciplinary spaces without sufficient support in terms of a community or recognized opportunities for such work. However, it is not just academics at the beginning of their careers who struggle with institutional rigidity. Established academics seeking career advancement are often assessed by colleagues and interdisciplinary work can sometimes be considered as less relevant, or in some extreme cases as not belonging to any field. Again, the consequence of this is that interdisciplinary intellectual curiosity can get relegated behind the security of familiar structures.

Similar obstacles can be encountered when applying for research grants or other forms of funding, and this obstacle to interdisciplinary work has been well documented (Huutoniemi et al., 2010; Jahn et al., 2012). Closely related is the difficulty of finding outlets for publication that welcome work crossing boundaries. The nature of academic publishing is that journals tend towards niches and specialization, and this can be highly discouraging for the interdisciplinary researcher. In the current highly competitive academic environment with its intense pressure to publish and secure research funds, it requires considerable courage to step outside familiar, "safe" structures. Although some research bodies are now claiming to support interdisciplinary work, it remains the responsibility of suitable reviewers to recognize the merits and validity of such work and this cannot always be guaranteed.

For individuals looking to pursue an interdisciplinary approach, an obvious practical constraint is the requirement to develop expertise in two or more disciplines. In the field of language learning psychology, this was a concern identified early on by Dörnyei (2005: 219), who recognized that "it is difficult to acquire sufficient expertise in two such different fields as linguistics and psychology". The risk with some interdisciplinary work is that rather than being creative, innovative and pushing boundaries, it can be simplistic and naive, ignoring work already conducted in both disciplines and failing to see the connections across subareas in each discipline. This can place an unrealistic burden on individual scholars who are required to be fully conversant with the literature in both applied linguistics and psychology to fully appreciate the complexity and connections within and across the fields.

The ideal scenario to cope with the problems posed by requiring expertise in multiple fields is to conduct studies integrating experts from the different fields. However, this fundamentally requires an openness and flexibility to working with others not only across disciplines but often epistemological and methodological boundaries as well as potentially practical institutional boundaries. Given some of the institutional constraints and pressures outlined above, it is understandable why some scholars may be reluctant to engage in such endeavours. Those coming from applied linguistics may find it easier, although by no means without problems, than those from psychology. As we have seen, applied linguistics has a history of interdisciplinarity and a stronger culture of methodological plurality. Whereas, in contrast, psychology is traditionally less open to other disciplines and approaches to research, pressure to conform to the established norms of the discipline is likely to be stronger.

\section{Possible agenda for promoting a new interdisciplinary field}

It is clear that each interdisciplinary endeavour faces different challenges defined by the nature of the disciplines involved (van Rijnsoever and Hessels, 2011) and these may also differ across settings. In the study of language learning psychology, the two core disciplines involved are applied linguistics and psychology. A clear first step in establishing interdisciplinary legitimacy is to attract interest and participation from scholars outside applied linguistics. Without such involvement, the new field cannot claim to be truly interdisciplinary. A good example of this in practice is Lou and Noels' (2016) recent study of mindsets in language learning in which they draw explicitly on both psychological and language learning related frameworks. What is perhaps most interesting about this work for us here is that the researchers chose to submit this to a mainstream educational psychology publication. More such cross-disciplinary publications are required to raise the profile within mainstream educational psychology of relevant and potentially ground-breaking work been conducted at the crossroads of applied linguistics and educational psychology.

To be recognized as a legitimate scholarly field and to encourage a sense of community, there is also a need for some degree of institutional formalization involving the development of bodies such as professional organizations, journals and conferences. At present, there has been a growth in published research as noted earlier this article, there is now an established biennial conference and there are several universities, which offer individual courses on the field as part of their programmes (see also Gkonou and Daubney, 2015). Here we conclude that it is incumbent on more established scholars to take the first steps to actively generate a community to which, although not exclusively, young scholars can come together, develop a sense of belonging and an identity as part of a larger body of people working in an area that is recognizable to those within as well as those beyond. Having an established professional association would help people to develop the confidence and community to support their work and to help them network and collaborate. The existence of an organized professional body also sends an important message to institutions and gatekeepers about the validity, strength and professionalism of the field.

A further step that needs to be taken is a serious reflection on quality criteria for work that is so overtly interdisciplinary. It is all very well to create new hybrid forms of research, but not at the expense of quality or academic rigour. New forms must combine and integrate insights but do so in a way that ensures that the work reflects the complex and nuanced understandings existing in established academic disciplines, not in a way that reduces or simplifies. These new forms should complement and add to existing forms, not detract from them. In the long term, guidelines for good practice in the field will be needed to support those starting out, reviewing for journals or acting as gatekeepers for funding bodies. Yet, it remains important that while establishing such professional criteria and organizations, we do not stifle the creativity, fluidity and innovation that is so clearly flourishing at present in the field. We want the field to gain recognition to facilitate and support its work but we do not want it to fall victim to restrictive, prescriptive rhetoric.

Finally, to develop as a legitimate interdiscipline, there needs to be more explicit discussion of our own interdisciplinarity. Genuine engagement with both the literature and wider community of interdisciplinarians can offer our emerging field both support and direction. The support comes from the knowledge that we belong to a wider community sharing a similar outlook and the direction comes from discussions that enable us to establish our own future aims and goals. 
Legitimacy and recognition do not come easily to newly emerging fields of study, especially those with an interdisciplinary character. The psychology and language learning community seeking to establish itself as a recognizable field of research must ensure that the nature and cultural tenets of interdisciplinarity remain prominent in its profile as it establishes itself and gains confidence. This means retaining an openness and a stance of inclusion, not exclusion. The field must remain self-aware and selfcritical, ensuring that those strengths of interdisciplinarity on which it is built define how it grows. From the perspective of the two authors, many of the challenges that face our field are difficulties that we have personally experienced in our own careers to date. The benefits of belonging to the active community that is emerging and being a part of some truly exciting work traversing disciplinary boundaries make those challenges worthwhile.

\section{References}

Boo Z, Dörnyei Z and Ryan S (2015) L2 motivation research 2005-2014: Understanding a publication surge and a changing landscape. System; 55: 145-157.

Breen MP (2001) Learner Contributions to Language Learning: New Directions in Research. Longman: Harlow, UK.

Campbell D (1969) Ethnocentrism of disciplines and the fish-scale model of omniscience. In: Sherif $\mathrm{M}$ and Sherif $\mathrm{C}$ (eds). Interdisciplinary Relations in the Social Sciences. Aldine: Chicago, IL, pp 328-348.

Cook G (2003) Applied Linguistics. Oxford University Press: Oxford.

Davis A (2007) An Introduction to Applied Linguistics. 2nd edn, Edinburgh University Press: Edinburgh.

Dörnyei Z (2005) The Psychology of the Language Learner: Individual Differences in Second Language Acquisition. Lawrence Erlbaum: Mahwah, NJ.

Dörnyei Z (2009) The Psychology of Second Language Acquisition. Oxford University Press: Oxford.

Dörnyei Z and Ryan S (2015) The Psychology of the Language Learner Revisited. Routledge: New York.

Fuchsman K (2012) Interdisciplines and interdisciplinarity: Political psychology and psychohistory compared. Issues in Integrative Studies; 30: 128-154.

Gkonou C and Daubney M (2015) Editorial. Humanising Language Teaching; 17(4), http://www.hltmag.co.uk/aug15/edit.htm.

Gkonou C, Tatzl D and Mercer S (eds) (2015) New Directions in Language Learning Psychology. Springer: Cham.

Huutoniemi K, Klein JT, Bruun H and Hukkinen J (2010) Analyzing interdisciplinarity: Typology and indicators. Research Policy; 39 (1): 79-88.
Jahn T, Bergmann M and Keil F (2012) Transdisciplinarity: Between mainstreaming and marginalization. Ecological Economics; 79: 1-10.

Lou M and Noels K (2016) Changing language mindsets: Implications for goal orientations and responses to failure. Contemporary Educational Psychology; 46: $22-33$.

MacIntyre P and Mercer S (2014) Introducing positive psychology to SLA. SSLLT; 4 (2): 153-172.

McDonough SH (1981) Psychology in Foreign Language Teaching. Allen \& Unwin: London.

Mercer S, Ryan S and Williams M (eds) (2012) Psychology in Language Learning: Insights from Theory and Research. Palgrave Macmillan: Basingstoke, UK.

Newell WH (2013) The state of the field: Interdisciplinary theory. Issues in Interdisciplinary Studies; 31 (2013): 22-43.

Ryan S and Mercer S (eds) (2015a) Studies in Second Language Learning and Teaching -Special Issue: Psychology in Language Learning 1; 5 (2).

Ryan S and Mercer S (eds) (2015b) Studies in Second Language Learning and Teaching -Special Issue: Psychology in Language Learning 2; 5 (3).

van Rijnsoever FJ and Hessels LK (2011) Factors associated with disciplinary and interdisciplinary research collaboration. Research Policy; 40(3): 463-472.

Widdowson $\mathrm{H}$ (2006) Applied linguistics and interdisciplinarity. International Journal of Applied Linguistics; 16 (1): 93-96.

Williams M and Burden RL (1997) Psychology for Language Teachers: A Social Constructivist Approach. Cambridge University Press: Cambridge, UK.

Williams M, Mercer S and Ryan S (2015) Exploring Psychology in Language Learning and Teaching. Oxford University Press: Oxford.

\section{Additional Information}

Competing interests: The authors declare no competing financial interests.

Reprints and permission information is available at http://www.palgrave-journals.com/ pal/authors/rights_and_permissions.html

How to cite this article: Mercer S and Ryan S (2016) Stretching the boundaries: language learning psychology. Palgrave Communications. 2:16031 doi: 10.1057/palcomms.2016.31.

(c) (i) This work is licensed under a Creative Commons Attribution 4.0 cc International License. The images or other third party material in this article are included in the article's Creative Commons license, unless indicated otherwise in the credit line; if the material is not included under the Creative Commons license, users will need to obtain permission from the license holder to reproduce the material. To view a copy of this license, visit http://creativecommons.org/licenses/by/4.0/ 